\title{
L'acceptabilité sociale des activités portuaires au Québec : vers une gouvernance territoriale? ${ }^{1}$
}

\author{
Yann Fournis ${ }^{2}$, Ousmane Mbaye ${ }^{3}$, Emmanuel Guy $^{4}$
}

\section{INTRODUCTION}

Si l'importance des ports dans le commerce international n'est plus à démontrer, leur capacité à s'insérer dans leur environnement territorial s'avère problématique. Confronté à un environnement international très concurrentiel, chaque port essaie de se positionner pour attirer investisseurs et compagnies, afin de se constituer en plaque tournante du commerce global. Cette mobilisation se manifeste au niveau local par la multiplication des projets de développement d'infrastructures portuaires, qui suscitent souvent des débats, des critiques voire des controverses au sein des populations locales. Ainsi, la diversification des activités et l'extension des zones d'exploitation portuaires posent souvent des problèmes d'acceptabilité sociale des projets portuaires - et en particulier dans un environnement fortement urbanisé, où ils connaissent de plus en plus « une insertion territoriale difficile » (Villeneuve, 2014). Les ports du Canada et du Québec sont au cœur de ces réalités à la fois internationales et locales. D'une part, les réformes importantes des années 1990 entendaient adapter l'organisation et le fonctionnement des ports aux nouvelles exigences internationales (concurrence, rentabilité économique) et locales (revendications des populations). D'autre part, au Québec comme ailleurs (Beuret, Cadoret, 2014), la multiplication des conflits et controverses indique la réelle difficulté de la gouvernance portuaire à se repositionner dans ses territoires.

Pour comprendre cette tension dans toute sa complexité, notre étude examinera la gouvernance portuaire, entendue comme une organisation hybride (i-e impliquant une multiplicité d'intervenants : public, privé, administration portuaire, société civile) faisant système (cf. Comtois, 2014 : 40-41) autour des activités de transport assurant l'interface entre les flux maritimes internationaux et les flux terrestres (société urbaine, arrière-pays et au-delà) (cf. Hall et Hesse, 2013). Plus précisément, notre objet est la recomposition des rapports entre territoires et activités portuaires durant des controverses localisées dans deux ports au Québec : le Port de Québec et le Port de Sept-Îles. Sur chaque site, deux controverses récentes (poussière rouge et silos à Québec; quai multiusager et déversement de mazout à Sept-Îles) ont servi de porte d'entrée pour comprendre l'inscription territoriale des acteurs portuaires, leur mode de gouvernance, leur capacité d'intervention, les problèmes auxquels ils sont confrontés et leurs relations avec l'environnement immédiat. Notre enquête, exploratoire et qualitative ${ }^{5}$, observe le déroulement des controverses pour comprendre comment les acteurs portuaires opèrent une redéfinition de leur mission traditionnelle d'interface entre flux globaux et réalité locale, et comment cette transformation des pratiques en vigueur et de la configuration des acteurs peut aboutir à une meilleure acceptabilité sociale des activités et projets portuaires.

\section{Notre objet est la recomposition des rapports entre territoires et activités portuaires durant des controverses \\ localisées dans deux ports au Québec : le Port de Québec et le Port de Sept-Îles.}

Notre analyse se propose, dans un premier temps, de mettre en exergue les évolutions récentes de l'administration portuaire et de son encadrement juridique. Après quelques décennies d'application de réformes qui ont abouti à des changements importants, les administrations portuaires sont appelées à s'adapter à un environnement (interne et externe) plus complexe et exigeant. Ensuite, à partir des cas des ports de Québec et de Sept-Îles, nous analyserons les 
processus d'émergence, de manifestation et les modes de régulation des controverses. Même si les cas présentés sont assez différents à première vue, une analyse fine permet de pointer des convergences. Nous terminerons par une synthèse analytique sur la gouvernance des ports et l'acceptabilité sociale des activités et des projets de développement portuaire pour montrer que les controverses font souvent l'objet d'une redéfinition de la vision commune d'un territoire et une recomposition des alliances entre les acteurs à différentes échelles.

\section{GOUVERNANCE PORTUAIRE AUX PRISES AVEC UNE DOUbLE CONTRAINTE INTERNATIONALE ET LOCALE}

Le tournant des années 1990 a été marquant dans le processus d'évolution des institutions portuaires au niveau international. Plusieurs réformes visent à adapter les organisations portuaires aux nouvelles réalités commerciales internationales comme, au Canada, la réforme de 1998 (révisant la Loi maritime) qui avait pour objectif de restructurer les ports canadiens afin de les doter d'une orientation plus commerciale et compétitive. Dans un contexte de restriction budgétaire peu porteur pour les exigences d'investissement des ports, le gouvernement fédéral ouvre la gouvernance portuaire au secteur privé, pour subvenir à ses besoins grandissants de financement. Entre libéralisation et privatisation, ce « quasi » retrait de l'état combiné à une promotion des acteurs privés transforme « la gestion institutionnelle des ports » (Comtois, Slack, 2003 : 11). L'objet de la Loi maritime du Canada fait ainsi état d'une volonté de mettre en place une politique marine permettant de renforcer la vitalité des ports (compétitivité internationale), adapter les infrastructures maritimes et les services avec les normes internationales, répondre aux besoins des utilisateurs, prendre en compte des besoins locaux et des questions environnementales.

\section{Notre étude s'est intéressée aux controverses} liées à des questions environnementales [...] et à la légitimité des arrangements autour des projets d'investissement.
Concrètement, les enjeux de compétitivité internationaux se matérialisent par des projets de réaménagement de l'espace portuaire, la diversification des activités et la construction de mégaprojets (quai multiusager, silos) qui vont questionner l'insertion des ports dans leur territoire. Si l'histoire des ports au Québec est intimement liée à leur ville d'implantation, il n'en demeure pas moins que les récents changements posent des problèmes de cohabitation qui complexifient la relation Ville-Port. Les projets de développement des autorités portuaires sont confrontés à diverses revendications des populations locales, qui reposent sur les problèmes «d'accès, de partage, d'utilisation, de gestion, de contrôle de ressources et espaces, ainsi que les oppositions liées à l'aménagement du territoire et aux enjeux géopolitiques qui y sont liés » (Beuret, Cadoret, 2014 : 211). Dans cette perspective, notre étude s'est intéressée aux controverses liées à des questions environnementales (avec les épisodes de «poussière rouge» au Port de Québec et le déversement de mazout dans la baie de Sept-Îles) et à la légitimité des arrangements autour des projets d'investissement (la question de la coopération pour la construction et l'accès du quai multiusager au Port de Sept-Îles; la question de l'aménagement et du paysage visuel pour les silos à Québec). Chaque conflit rend compte d'un processus conflictuel ${ }^{6}$ particulier en fonction des acteurs (publics, privés) impliqués et des enjeux sociaux environnementaux et économiques en jeu.

\section{LA CONFIGURATION DES ACTEURS FACE AUX CONTROVERSES}

L'existence de controverses sur les deux sites (Québec et Sept-Îles) indique que le secteur portuaire n'est pas resté à l'écart des grands débats social, économique et surtout environnemental animant la vie économique canadienne : conformément à leur vocation historique (liée aux «staples »: Watkins, 2007). Les deux ports consacrent la part principale de leurs activités aux matières premières. Si cette spécialisation ne va plus de soi dans un contexte d'épuisement du mode traditionnel d'exploitation des ressources, elle demeure une stratégie courante dans le monde portuaire contemporain. Toutefois, cette explication, simple, n'explique pas vraiment pourquoi, 
suite aux récentes réformes opérées dans la plupart des grands ports du monde, les administrations portuaires font souvent face à des problèmes de cohabitation avec leur milieu (Villeneuve, 2014). Pour comprendre cette tendance, il convient d'affiner le grain d'observation, en menant une analyse longitudinale des controverses, pour saisir comment chacune d'entre elles a bousculé à la fois les pratiques en vigueur et la configuration des acteurs en présence.

\subsection{Les controverses au Port de Québec}

Le Port a d'abord été l'objet d'une
polémique d'ampleur, suite à la découverte
d'une « vague » de poussière rouge dans les
quartiers environnants, à proximité de ses
installations.

Le Port de Québec est une institution, à la fois le plus vieux port du Canada et sa deuxième administration portuaire (en tonnage manutentionné), qui se positionne comme un important joueur dans le système de transport canadien et international (avec surtout une spécialisation dans les vracs solide et liquide). Mais, l'actualité récente a surtout rappelé la fragilité de son insertion locale, menacée par une série de controverses entourant aussi bien les activités quotidiennes du port que ses projets d'agrandissement.

Pour ce qui concerne ses activités régulières, le port a d'abord été l'objet d'une polémique d'ampleur, suite à la découverte d'une « vague » de poussière rouge dans les quartiers environnants, à proximité de ses installations (octobre 2012). Des opérations de transbordement réalisées par l'entreprise Arrimage Québec soulèvent des suspicions de la part des populations. Inquiets, des habitants du quartier Limoilou commandent une étude auprès d'un laboratoire privé afin d'en savoir plus sur cette «poussière rouge » - dont les résultats, surprenants quant à la charge de métaux lourds ${ }^{7}$, déclenchent une mobilisation citoyenne. Autour de quelques leaders, se cristallisent les revendications des populations qui demandent des explications à l'administration portuaire et à son partenaire Arrimage Québec et sollicitent le gouvernement du Québec (pour vérification du respect des normes environnementales provinciales).
Confrontées aux critiques citoyennes et aux injonctions réglementaires de Québec en matière environnementale, les autorités portuaires tentent maladroitement de se défendre, avançant qu'elles ne dépendent pas de la juridiction provinciale concernant les questions environnementales. Plus encore, Arrimage Québec refuse un temps de reconnaître les résultats obtenus par les citoyens en arguant de ses propres tests, rassurants ${ }^{8}$. Cette phase démontre que l'objet même de la controverse est mal identifié, poussant à un usage politique de la connaissance scientifique (études) et de la technologie (système de contrôle et de protection de la poussière) afin de répondre à la controverse naissante. Or, cet usage provoque rapidement un durcissement des positions de part et d'autre: la phase, très conflictuelle, est marquée par les accusations portées à l'encontre du port et Arrimage Québec. Or, les tentatives de démonstration scientifique ne parviennent pas à dépolariser le conflit ${ }^{9}$ et s'achèvent par des procédures judiciaires (avis de non-conformité, dépôt d'un recours collectif), qui dénotent elles-mêmes des interprétations différentes des lois régissant les milieux portuaires et les compétences respectives des instances politiques provinciales et fédérales sur les questions environnementales.

Le déblocage de la controverse viendra d'une recomposition plus générale du système d'acteurs, avec la mise en place des tables de concertation mixtes regroupant les principaux protagonistes (Comité de vigilance des activités portuaires, Comité intersectoriel pour la contamination environnementale dans la cité de Limoilou, Comité des relations avec la communauté). Espaces neutres de dialogue et de concertation, ces comités laissent augurer une nouvelle phase, basée sur la négociation voire la coconstruction de l'objet de la controverse, dans des nouvelles structures élargissant la gouvernance portuaire traditionnelle. Ces nouveaux arrangements organisationnels pourraient traduire une sortie vers le haut de la controverse, vers la définition d'une vision commune du développement territorial. Dans ce sens, on pourra dire que les conflits portuaires peuvent instituer «une forme de territorialisation parce qu'ils produisent des groupes, conscients d'une solidarité de destin reposant sur leur inscription spatiale, qui s'approprient matériellement, par 
une veille de terrain, et idéalement, par un travail de nomination et de valorisation, un espace » (Melé, 2008 : 16).

Une seconde controverse a touché le Port de Québec, ou plus spécifiquement l'un de ses projets d'agrandissement: la construction de silos pour le stockage de granules de bois. L'apparition du premier dôme (15 novembre 2013) suscite des critiques de la part des riverains qui évoquent le caractère imposant de la structure, ses «effets visuels » et diverses nuisances (bruit, poussière) ou risques (d'incendie) ${ }^{10}$. Même si le port a organisé une séance de consultation avant le début de la construction, les habitants dénoncent la communication unidirectionnelle des dirigeants du port (absence d'information plus détaillée, non prise en compte de leurs préoccupations) et affichent leurs réticences lors de la poursuite des travaux. Ceci va susciter un durcissement des positions et une polarisation du conflit. D'un côté, les autorités portuaires ainsi que l'entreprise privée (Arrimage Québec) constituent une coalition pour le développement portuaire et la poursuite des travaux de construction; pour eux, le respect des normes environnementales fédérales est suffisant pour aller de l'avant dans la réalisation du projet. D'un autre côté, les citoyens et le gouvernement du Québec réfutent catégoriquement cette justification : la mobilisation s'étend, d'abord à certains conseils de quartier, puis à des organisations écologiques (Les Amies du Saint-Laurent, Nature Québec et le Conseil régional de l'environnement) qui considèrent le projet des silos comme un «mauvais projet au mauvais moment ${ }^{11}$.

Comme pour la controverse de la poussière rouge, le dénouement du conflit viendra d'un élargissement simultané de l'enjeu controversé et des acteurs conviés à le traiter, suite à la création d'un comité pour l'embellissement des silos. Chargé de réfléchir sur une meilleure manière d'intégrer la structure dans son environnement, son apport sera modeste (il suggère de laisser l'apparence blanchâtre telle quelle), mais permettra de dissiper les tensions et de poursuivre les travaux pour le second silo.

Ces deux cas indiquent que le Port de Québec a connu des conflits sévères ces dernières années, touchant aussi bien ses nouveaux développements que ses activités traditionnelles, mais que l'existence de comités de concertation a permis de rapprocher fructueusement les positions et d'insérer plus étroitement les dynamiques portuaires dans son environnement territorial.

\subsection{Les controverses au Port de Sept-îles}

Le Port de Sept-Îles présente a priori un profil très différent de son homologue de Québec: l'on pourrait s'attendre à ce que l'insertion territoriale d'un port minéralier dans une "régionressource » peu urbanisée se fasse sans heurt. Or, ici aussi les activités routinières et les projets de développement du port suscitent des conflits d'usage de l'espace, indiquant l'acuité de la question environnementale et la fragilité de la gouvernance portuaire.

\section{Des opérations de manutention de l'entreprise Cliffs Natural Resources occasionnent un déversement d'une quantité importante de mazout.}

La première controverse observée est liée aux activités routinières du port, ou plutôt aux effets de débordement que provoque un incident : dans la nuit du 30 au 31 août 2013, des opérations de manutention de l'entreprise Cliffs Natural Resources occasionnent un déversement d'une quantité importante de mazout. L'ampleur ${ }^{12}$ de ce qui est considéré par l'entreprise comme un accident ne va pas manquer de susciter une grogne au sein de la population. La controverse est initiée par des critiques virulentes dans les médias, issues de la population septilienne, pour dénoncer (ce qui est considéré comme) une négligence de la part de l'entreprise, ainsi que le manque de transparence dans la gestion de l'après-crise. Toutefois, le répertoire technique et administratif classique de la gouvernance portuaire a été très rapidement et efficacement mobilisé : l'enjeu étant bien identifié, l'entreprise a très tôt pris les mesures nécessaires pour endiguer le phénomène, sous la supervision des agents du ministère de l'Environnement, et a organisé une journée porte ouverte. De même, les mécanismes classiques de la Justice aboutissent pour établir les responsabilités: une enquête 
diligentée par le gouvernement provincial aboutit quelques mois plus tard à une condamnation de l'entreprise ${ }^{13}$. Retenons donc de cette crise le fait que la controverse ne donne pas vraiment lieu à une polarisation poussée: si l'on assiste effectivement à des prises de position de la part d'associations ou de collectifs $^{14}$, celles-ci se résorbent rapidement, tandis que la gouvernance portuaire apparaît en mesure de gérer l'enjeu de la pollution. Il en va différemment pour la controverse autour du quai multiusager.

Au titre des projets de développement portuaire, l'enjeu de la construction d'un quai multiusager est considéré par le port comme vital pour se positionner sur l'échiquier du transport maritime international, grâce à un partenariat public-privé innovant ${ }^{15}$. Si le dossier est longtemps consensuel (phases de recherche de partenaire et du début des travaux), il va déraper brutalement vers la fin des travaux, mettant à jour la fragilité de la gouvernance portuaire. Tout se joue autour de l'enjeu crucial de l'accessibilité aux équipements pour les partenaires privés du projet, sur laquelle l'entreprise privée Cliffs possède de facto une sorte de veto ${ }^{16}$. Or, loin d'aboutir, les négociations entre le port et Cliffs pour la vente des terrains et l'accessibilité du chemin de fer s'enferment dans une impasse - ce qui crée une situation un peu ubuesque pour les cinq entreprises minières ayant cofinancé le projet... et qui n'ont pas accès au quai faute d'entente signée avec Cliffs. Rapidement, les intérêts s'affrontent : tandis que Cliffs dépose une plainte contre le Port (lui reprochant de retarder le début de la réfection au quai 30 pour lui forcer la main), le port sollicite l'Office des transports du Canada afin de réaffirmer le statut du chemin de fer reliant la ligne principale au nouveau quai multiusager en construction. Le conflit s'élargit encore lorsque les partenaires financiers du projet et une organisation locale réclament le statut d'intervenant pour plaider (en faveur de l'administration portuaire) puis lorsque le gouvernement provincial mandate trois ministres pour rouvrir les négociations - qui, en dépit d'un ultimatum, ne permettent pas de dénouer la crise. Le déblocage viendra finalement de l'intervention du gouvernement fédéral qui, après avis de l'Office du transport, qui impose aux propriétaires du chemin de fer, reconnu d'intérêt national, d'offrir le service de transport à tous les expéditeurs souhaitant y faire transiger leurs marchandises tel que le prévoit la Loi sur les transports au Canada.

Observons donc que ce dossier a surtout mis en valeur la fragilité interne de la gouvernance portuaire, dont le fonctionnement est bloqué par la division de la coalition portuaire qui, du fait du flou de la répartition formelle des compétences et des équilibres de pouvoir, doit faire appel à des instances extérieures pour mettre en œuvre son nouveau projet de développement.

Le Port de Sept-Îles a donc connu, lui aussi, des controverses relatives à ses activités routinières ou à ses nouveaux développements. Certes, le fait d'être localisé dans une «région-ressource» facilite peut-être ses relations avec son environnement, mais pas au point d'éviter toute tension en cas de débordement ou en cas de conflits internes à la coalition portuaire. En fait, si les controverses étudiées paraissent plus facilement gérables, cela semble surtout tenir au fait qu'elles visent moins à une contestation du répertoire portuaire traditionnel qu'à des tensions entourant sa mise en œuvre. Bref, les objets sociotechniques et ceux qui les traitent ne sont en l'occurrence pas les mêmes qu'à Québec. Toutefois, le conflit autour du quai multiusager met à jour une autre fragilité de la gouvernance portuaire qui, en cas de conflit interne, ne peut débloquer les dossiers qu'en recourant à un arbitrage externe permettant une redéfinition radicale des termes de la controverse (changement de statut juridique de la voie de chemin de fer).

\section{LA GOUVERNANCE PORTUAIRE FACE AU DÉFI DE L'ACCEPTABILITÉ SOCIALE : UNE PERSPECTIVE TERRITORIALE}

Les controverses examinées confirment que la position traditionnelle des ports, comme interface entre «leur»société territoriale et leur environnement global, est actuellement l'objet de fortes tensions. Celles-ci peuvent être analysées à partir de la notion d'acceptabilité sociale, définie comme «un processus d'évaluation politique d'un projet sociotechnique mettant en interaction une pluralité 
d'acteurs impliqués à diverses échelles et à partir duquel se construisent progressivement des arrangements et des règles institutionnels reconnus légitimes, car cohérents avec la vision du territoire et le modèle de développement privilégié par les acteurs concernés » (Fournis, Fortin, 2013 : 13). Cette définition, large, a l'intérêt d'englober les différentes dimensions qui apparaissent comme centrales dans la construction des territoires (cf. schéma). Reprenons chacune de ces dimensions, pour observer qu'elles sont complémentaires plus qu'opposées pour saisir les controverses mettant en jeu l'acceptabilité territoriale des activités et projets portuaires.

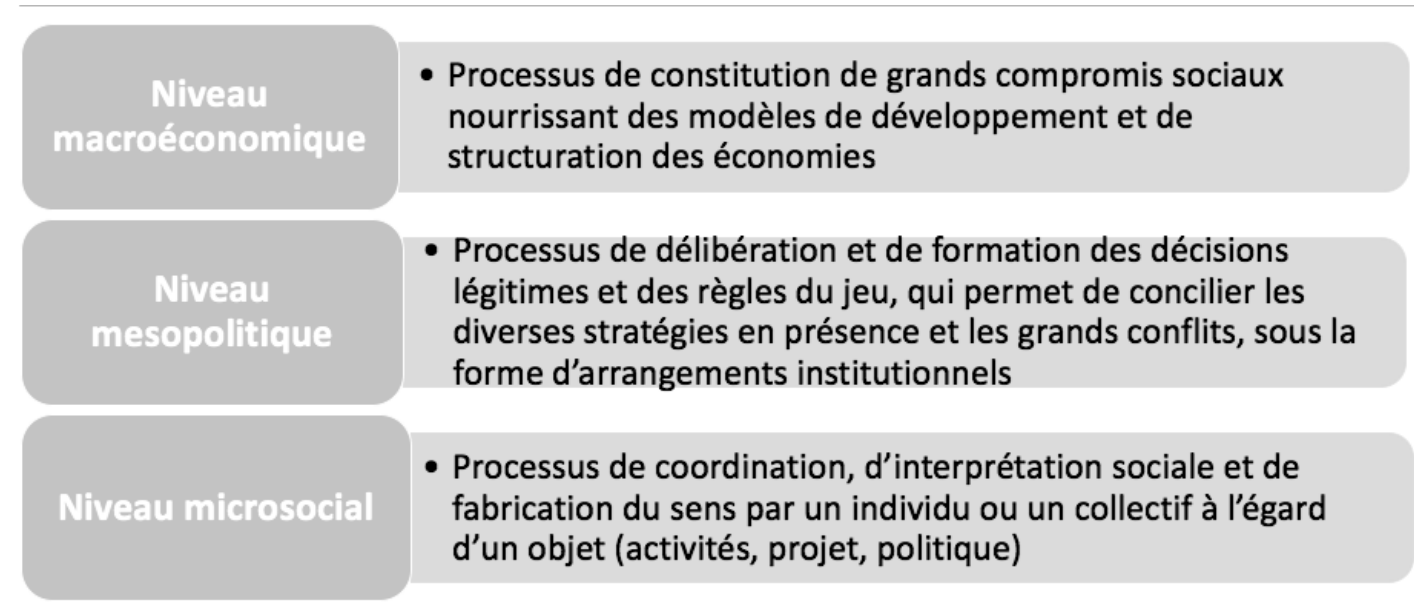

Fortin, Fournis, 2013

\section{L'existence de controverses confirme que, au niveau macroéconomique, les rapports entre activités économiques traditionnelles et sociétés territoriales se sont profondément transformés.}

Dans une certaine mesure, l'existence de controverses confirme que, au niveau macroéconomique, les rapports entre activités économiques traditionnelles et sociétés territoriales se sont profondément transformés. Ceci valide notamment les analyses sur la transition économique du Canada, qui passerait d'un modèle stable d'exploitation des ressources (de type staple), à un modèle plus complexe et contesté, où les activités liées aux ressources naturelles n'auraient plus leur évidence passée (modèle dit post-staple). La controverse autour des silos du Port de Québec s'explique assez bien en ces termes : la vocation industrielle traditionnelle de l'espace concerné est remise en cause par de nouvelles revendications, témoignant des transformations de la structure sociale de la ville, en voie de gentrification. Toutefois, ce type d'explication globale présente rapidement des limites lorsqu'il est question de la controverse autour de la «poussière rouge » à Québec et surtout les conflits entourant le Port de Sept-Îles : ils touchent moins la dimension symbolique du développement portuaire (le paradigme de développement) que le fonctionnement de la gouvernance portuaire elle-même et sa capacité à traiter correctement certains enjeux sociotechniques pourtant traditionnels (pollution, projet de développement). Pour saisir ces différentes dynamiques, il faut affiner le grain de l'analyse pour observer deux types de tension : celles qui, au niveau mésopolitique, bousculent les arrangements politiques assurant le fonctionnement régulier du port (la gouvernance portuaire) et celles qui témoignent, à un niveau micro-social, de l'épuisement du répertoire administratif et technologique à gérer efficacement les enjeux portuaires.

\subsection{La gouvernance portuaire : entre} contestations et élargissement mésopolitique

Sous une perspective mésopolitique, notre enquête aboutit d'abord à souligner que l'opposition entre la population locale et la coalition 
portuaire est loin d'être le principal facteur de tension autour de la gouvernance portuaire. En effet, son fonctionnement apparaît comme complexe et fragile, parce qu'il exige à la fois un consensus interne et une ouverture constante à son environnement extérieur. Cette complexité est d'abord liée au statut juridique ambigu des installations portuaires, qui ouvrent à des évaluations contradictoires sur les compétences des deux niveaux de gouvernement à légiférer sur les questions portuaires. Si le cas de Québec est évident (puisque les gouvernements fédéral et provincial eux-mêmes sont divisés), il est aussi patent à Sept-Îles où la controverse sur le quai multiusager donne lieu à l'intervention simultanée des pouvoirs publics de niveaux différents. Mais cette complexité apparaît surtout comme une source de fragilité lorsque les équilibres internes à la coalition portuaire débouchent sur un conflit - comme à Sept-Îles, où il faut rien moins que l'intervention de l'Office du transport pour débloquer un dossier qui recueille pourtant le soutien de la quasi-totalité des acteurs concernés. Observons de même que, dans ce contexte de blocage entre niveaux et entre types d'acteurs (public et privé), la voie judiciaire est systématiquement utilisée parce qu'elle seule semble en mesure d'opérer un arbitrage légitime. Bref, l'essor de contestations externes de la gouvernance portuaire est sans doute une tendance récente marquante, mais elle vient surtout mettre en évidence les faiblesses internes d'un univers fonctionnant au consensus nécessaire dans un contexte flou et contradictoire.

\section{L'essor de mobilisations au sein de la société locale peut être redoutable lorsqu'elles identifient un objet instable (cf. infra) et trouvent des relais dans l'espace public.}

Cette fragilité structurelle explique d'ailleurs l'impact des contestations externes sur la gouvernance portuaire. L'essor de mobilisations au sein de la société locale peut être redoutable lorsqu'elles identifient un objet instable (cf. infra) et trouvent des relais dans l'espace public, comme l'illustrent les épisodes de «poussière rouge » à Québec, qui mettent moins aux prises «le » port et «les » habitants, que la coalition portuaire d'une part et les acteurs sociaux et provinciaux d'autre part. Mais les mobilisations externes peuvent aussi être très marginales, lorsque l'enjeu sociotechnique est rapidement identifié et pris en charge efficacement par les autorités compétentes (ainsi du cas de déversement à Sept-îles). Ceci met d'autant plus en valeur l'importance $\mathrm{du}$ consensus et de sa fabrication: si le consensus apparaît comme une nécessité pour le bon fonctionnement interne de la gouvernance portuaire, il en va de plus en plus de même quant aux rapports du port avec son environnement. En témoigne le cas du port de Québec, qui s'enferme un temps dans la dénégation des effets de débordement de ses activités et projets, suscitant des controverses remarquables... avant d'enclencher une dynamique d'élargissement de la concertation à de nouveaux acteurs qui permettent assez efficacement de résorber les tensions avec les acteurs sociaux (ainsi du cas des silos). Sous cette perspective, il faudra sans doute que les acteurs portuaires envisagent que, si le consensus n'est pas une condition formellement indispensable à la gouvernance portuaire, elle est une nécessité à la fois interne et externe - sauf à accepter de confier le sort de la gouvernance des ports aux autorités judiciaires. Mais ces conclusions quant à la gouvernance portuaire dépendent aussi de la nature de l'enjeu sociotechnique concerné.

\subsection{Les enjeux sociotechniques : entre controverses et complexité microsociale}

Dans une perspective microsociale, la définition sociotechnique des enjeux prend une grande importance pour saisir le développement des controverses portuaires. Pour le dire simplement, une controverse basée sur un objet stabilisé par une expertise opérationnelle forte et non contestée est aussi en l'occurrence une controverse qui connaît peu d'effets de débordement et reste gérée dans les limites classiques de la gouvernance portuaire. À l'opposé, une controverse dont l'objet est peu stabilisé du fait de la remise en cause de l'expertise portuaire est aussi une controverse qui déborde sur la société locale, avec l'irruption d'acteurs sociaux (formels ou non) et d'acteurs politiques de différents niveaux. 
Ainsi, la «nature » des controverses sociotechniques influe fortement sur la capacité des acteurs portuaires à y faire face avec leurs outils traditionnels. Les cas de Sept-Îles et de Québec sont ici très contrastés.

Dans le premier cas, les deux controverses se cristallisent autour de phénomènes assez bien définis en termes techniques, financiers et stratégiques, qui n'entraînent pas de remise en cause de la répartition traditionnelle des rôles des différents acteurs - personne ne conteste la portée technique des enjeux (pollution, modernisation des installations), ce qui facilite considérablement la résolution de la controverse. À Québec au contraire, les controverses se nouent autour d'objets instables, aux frontières et à la réalité floues, qui suscitent de fortes inquiétudes dans la société locale et déstabilisent fortement la capacité de la gouvernance portuaire à les traiter. Ainsi, la définition du projet des silos échappe rapidement à ses initiateurs, suscitant une configuration incertaine, éclatée et fluide sur fond d'incertitudes quant à ses effets environnementaux et sociaux sur la société environnante. La controverse liée à la «poussière rouge » est plus spectaculaire encore: la gouvernance portuaire et de son répertoire opérationnel sont débordés par une définition alternative de l'objet de la controverse (aux causes et effets incertains, conflictuels), portée d'abord par des contestations citoyennes puis par les autorités provinciales. Après une période d'opposition stérile qui conduit à une forte polarisation, la configuration se débloquera par un élargissement de la gestion des débordements sociotechniques, conduisant à une définition plus ouverte de l'enjeu concerné. Retenons donc de ces controverses que, lorsque l'enjeu semble prouver l'incapacité du répertoire traditionnel de la coalition portuaire à gérer

\section{CONCLUSION}

Analyser l'acceptabilité sociale de la gouvernance portuaire au Québec conduit nécessairement à s'interroger sur les effets des changements institutionnels survenus dans les administrations portuaires ces dernières années. Comme la plupart des ports du monde, les ports du Québec (et du Canada) ont connu des réformes importantes, qui se traduisent dans une correctement ses débordements sur la société locale, il n'est sans doute pas très efficace de s'appuyer sur ce même répertoire pour disqualifier les critiques relatives aux effets de débordement des activités portuaires. Cependant, lorsque l'enjeu concerné est stabilisé par des paramètres techniques et administratifs traditionnels (certitudes techniques, routine administrative, maîtrise des risques), les critiques externes, issues de la société locale, sont moins importantes que le bon déroulement des rapports internes à la gouvernance portuaire.

\section{Il nous semble que la notion d'acceptabilité sociale éclaire bien les nouveaux enjeux de l'inscription territoriale de la gouvernance portuaire au Québec.}

De manière générale, il nous semble que la notion d'acceptabilité sociale éclaire bien les nouveaux enjeux de l'inscription territoriale de la gouvernance portuaire au Québec. Ainsi, celle-ci concerne différentes dimensions (macroéconomiques, mésopolitiques et sociotechniques), que l'on peut difficilement séparer ou rabattre sur un seul type d'enjeu (par exemple, la gentrification de la ville de Québec). Si ces différentes problématiques prennent des formes différentes selon les controverses et les territoires, il nous semble qu'elles renvoient à une tendance générale: dans un contexte de fragilisation des activités portuaires liées à l'exploitation traditionnelle des ressources naturelles, la gouvernance portuaire est effectivement de plus en plus confrontée aux limites de son répertoire usuel pour traiter des objets sociotechniques instables, peu définis et complexes. Ceci n'est pas sans questionner le fonctionnement de la gouvernance portuaire.

recomposition (très) peu lisible : l'impératif de développement, de diversification des activités portuaires, l'autonomisation des administrations et la réduction des financements publics ont contraint les ports à adopter des modes d'organisation et de fonctionnement nouveaux, exigeants et fragiles alors même que s'accroissaient les contraintes externes, interna- 
tionales (avec une compétition accrue) et locales (avec l'essor de mobilisations critiques évoquant une « vigile » permanente). Or, la multiplication récente des controverses, qui incarnent toutes cette articulation entre le local et l'international, rend autant compte de la défaillance des compromis anciens que des difficultés à nouer un dialogue territorial fructueux.

\section{Analyser l'acceptabilité sociale de la gouvernance portuaire au Québec conduit nécessairement à s'interroger sur les effets des changements institutionnels survenus dans les administrations portuaires ces dernières années.}

Soulignons combien les controverses ont d'abord donné lieu à un dialogue de sourds, opposant des stratégies (comme à Sept-Îles) ou des argumentaires inconciliables (la demande du marché international/les questions sociale, économique et surtout environnementale à Québec). Ceci illustre deux traits fondamentaux de la gouvernance portuaire, qui ne peut fonctionner en l'état actuel que sur une logique de «partenariat obligatoire» entre acteurs portuaires (dont l'enrayement est redoutable, comme pour le quai multiusager à Sept-Îles) et une logique de «partenariat optionnel » avec les acteurs locaux (qui se traduit parfois par un refus pur et simple de négocier qui, dans le flou juridique quant aux compétences des niveaux de gouvernement, s'achève nécessairement par une judiciarisation). Or, ce partage dichotomique favorisé par les réformes portuaires apparaît de moins en moins tenable, soit parce qu'il crée des controverses difficiles, soit (plus intéressant) parce qu'il est rendu obsolète par des pratiques innovantes. Des progrès semblent ainsi se faire jour à Québec, suite à la mise en place de cadres de concertation multipartite ${ }^{17}$ qui dévoilent « des visions du territoire, mais aussi des attentes, des argumentations sur les modalités de l'insertion et la position spatiale occupée par certains habitants » face aux projets et activités portuaires (Melé, 2004). Sous cette perspective, il y aurait au-delà de chaque conflit une tendance à la redéfinition de la territorialité des activités portuaires, passant par une recombinaison des alliances et des structures politiques de concer- tation qui pourraient, en hypothèse, conduire à une requalification des enjeux sociotechniques. Il $\mathrm{y}$ a donc lieu d'adopter un ton modérément optimiste. Certes, ceci n'est pas une panacée, ne serait-ce que parce que cela participe de la confusion et de l'opacité de la gouvernance portuaire (comme le précise une citoyenne de Québec : «il y a beaucoup de comités, là. Sans vouloir faire de jeu de mots, il faut arrimer tout ça ${ }^{18}$ ). Mais cela indique aussi que, plus qu'un dysfonctionnement fondamental de la gouvernance portuaire, les controverses portuaires constitueraient un moteur de la redéfinition des rapports entre le port et le territoire.

\section{BIBLIOGRAPHIE}

Beuret, J.E., Cadoret, A. (2014). «De l'analyse des conflits à l'étude des systèmes conflictuels : l'exemple des conflits environnementaux et territoriaux dans les trois plus grands ports maritimes français (Marseille-Fos, Le Havre et Dunkerque)», Géographie, économie, société, vol. 16, n 2 , pp. 207-231.

Cadoret, A. (2011). «Analyses des processus conflictuels », L'Espace géographique, 3, Tome 40, pp.231-244.

Comtois, C. (2014). «Les échelles géographiques à la gouvernance ville-port », in Y. Alix, B. Delsalle et C. Comtois (dir.) Port-city governance, Le Havre, Fondation Sefacil, pp. 39-54.

Comtois, C., Slack, B. (2003). «Innover l'autorité portuaire au $21^{\mathrm{e}}$ siècle: un nouvel agenda de gouvernance », Les Cahiers Scientifiques du Transport, $\mathrm{n}^{\circ} 44$, pp. 11-24.

Debrie, J., Ruby, C. (2009). Approche des réformes portuaires européennes et nord-américaines (Canada) : Éléments de réflexion pour la politique portuaire française, INRETS, $50 \mathrm{p}$.

Fournis Y. et Fortin M.-J., (2013). L'acceptabilité sociale de l'énergie éolienne: une définition, Rimouski, GRIDEQ | document de travail, $\mathrm{n}^{\circ} 131017$.

Hall, P.V., Hesse, M. (2013). Cities, Regions and Flows, Abingdon, Oxon ; New York, NY, Routledge, 270 p.

Melé, P. (2004). «Introduction : Conflits, territoires et action publique », in Melé, P., Larrue, C., \& Rosemberg, M. (dir.), Conflits et territoires, Tours, Presses universitaires François-Rabelais.

Melé, P. (2008). Conflits et controverses : de nouvelles scènes de production territoriale?, in Isabelle Garat, Raymond Séchet, Djémila Zeneidi, Espaces en (trans) action, Rennes, Presses Universitaires de Rennes, pp. 239-250.

Villeneuve, P. (2014). «Le Port de Québec comme mégaprojet: Une insertion territoriale difficile», Organisations et territoires, vol. $23, \mathrm{n}^{\circ} 3$, pp. 49-53. 
Watkins, M. (2007). «Staples Redux», Studies in Political Economy, vol. 79, p. 213-226.

\section{NOTES}

${ }^{1}$ Cette enquête a été réalisée entre mars 2014 et février 2015, pour le compte du MTQ. Nous remercions nos partenaires pour les critiques constructives qui ont été faites durant sa réalisation.

${ }^{2}$ Yann Fournis est professeur à l'UQAR et membre du Centre de recherche sur le développement territorial (CRDT) et du GRIDEQ (Groupe de recherche interdisciplinaire sur le développement régional, de l'Estdu-Québec).

${ }^{3}$ Ousmane Mbaye est étudiant dans le programme conjoint de doctorat en Développement régional UQAR-UQAC et membre du CRDT (Centre de Recherche sur le Développement Territorial).

${ }^{4}$ Emmanuel Guy est professeur à l'Unité départementale des sciences de la gestion du campus de Rimouski à l'UQAR et titulaire de la Chaire de recherche en transport maritime. Il est membre du GRIDEQ (Groupe de recherche interdisciplinaire sur le développement régional, de l'Est-du-Québec)

${ }^{5}$ Les données sont recueillies à partir d'une revue de presse basée sur les publications du journal Le Soleil entre juillet 2012 et janvier 2014, avec une base de données composée de 165 articles pour le Port de Québec et 43 pour le Port de Sept-Îles. L'objectif était de choisir des faits récents afin de déterminer des paramètres pertinents pour l'analyse de la dynamique de la gouvernance portuaire. L'observation porte sur une période allant de 2012 à 2015.

${ }^{6}$ Le conflit est défini ici comme une opposition formelle entre des acteurs organisés (Foulquier, 2009). Il s'inscrit dans un processus (Cadoret, 2011) qui lui imprime une dynamique (plus ou moins forte) dans le temps.

${ }^{7}$ Les résultats de l'étude avaient décelé les quantités suivantes de métaux sur les échantillons prélevés : Nickel : $474 \mathrm{mg} / \mathrm{kg}$, Zinc : $998 \mathrm{mg} / \mathrm{kg}$, cuivre : $664 \mathrm{mg} / \mathrm{kg}$

${ }^{8}$ Selon ces résultats, «aucun des métaux analysés dans l'échantillon n'excède la concentration maximale pour un terrain à vocation résidentielle ». Cf. Morin, A. «L'entreprise montrée du doigt se défend », Le Soleil, 20 décembre 2012.

${ }^{9}$ La présence ou non de métaux lourds dans l'environnement et leurs éventuelles conséquences sur les populations touchées est l'objet d'études par chaque groupe d'acteurs qui étaye ses arguments, mais n'est pas convaincu par les données de l'adversaire.

${ }^{10}$ Morin, A. «Un projet qui sème l'inquiétude », Le Soleil, 17 juillet 2013.

${ }^{11}$ Dans un communiqué commun, ils exigent l'arrêt immédiat des travaux afin de dégager le temps nécessaire à l'engagement des discussions en vue d'une étude environnementale indépendante. Cf. Morin, A. «En guerre contre le nouveau terminal », Le Soleil, 7 novembre 2013.

${ }^{12} \mathrm{La}$ fuite est estimée à quelque $5 \quad 000$ litres d'hydrocarbures, sur une surface de 7 kilomètres à partir des installations de l'entreprise.

${ }^{13} \mathrm{Si}$ l'entreprise refuse dans un premier temps d'admettre sa culpabilité, elle finit par reconnaître avoir enfreint la loi québécoise sur la qualité de l'environnement et accepte de payer un dédommagement à hauteur de $821000 \$$.
${ }^{14}$ Des organisations écologiques comme le regroupement pour la sauvegarde de la grande baie de Sept-Îles soulignent la vulnérabilité de la baie et dénoncent les risques de pollution environnementale liée à la manutention d'hydrocarbures dans le Port de Sept-îles. De son côté, la mobilisation citoyenne s'est organisée spontanément à travers la signature d'une pétition et, par la suite, le dépôt d'une requête auprès du ministère de l'Environnement pour la tenue d'enquête publique. Cf. notamment Lévesque F. «Le déversement de mazout dans la balance », Le Soleil, 21 septembre 2013.

${ }^{15}$ Le projet, à hauteur de 220 millions de \$, est cofinancé à $50 \%$ par le public avec une contribution de $25 \%$ pour le gouvernement fédéral et $25 \%$ par l'administration portuaire de Sept-Îles et, l'autre moitié est partagée entre cinq entreprises privées (New Millenium, Champion Minerals, Alderon Iron, Labrador Iron Mines, Tata Steel Minerals Canada).

${ }^{16}$ Rappelons que les terrains en amont du mégaquai en construction appartiennent à l'entreprise et que le seul chemin de fer donnant accès au futur quai est sa propriété privée.

${ }^{17}$ Les espaces de concertation mis en place: Comité de vigilance des activités portuaires, Comité intersectoriel pour la contamination environnementale dans la Cité-Limoilou, Comité des relations avec la communauté, comité pour l'intégration des silos dans le Port de Québec.

${ }^{18}$ Morin A. « Et trois comités », Le Soleil, 08 mai 2013. 